\title{
О ПОНИЖЕНИИ РАЗМЕРНОСТИ В ЗАДАЧАХ ОПТИМАЛЬНОГО СИНТЕЗА НЕКОТОРЫХ ДИНАМИЧЕСКИХ СИСТЕМ
}

Рассматривается задача оптимального синтеза $n$-мерной динамической системы, линейной по управлению. Нестационарная область управления, заданная системой неравенств, аппроксимируется одним ограничением. Для системы с информативными переменными задача сведена к решению характеристического уравнения размерности $l \ll n$ и выбору матрицы управления. Предложен новый способ решения проблемы Калмана-Летова - обращение посредством аппроксимации гиперкуба управления. Получено решение задач оптимального синтеза для $y$-инвариантных, $y$-одномерных и некоторых диссипативных систем.

1. Рассмотрим линейную по управлению $u$ динамическую систему

$$
\xi^{*}=X(q)+A_{1}(q) u, \quad X(t, 0) \equiv 0, \quad q=\left(t, \xi_{v}\right)^{*}, \quad X=\left(X_{v}\right)^{*},
$$

$$
\xi=\left(\xi_{\alpha}^{\prime}, \xi_{\beta}^{\prime \prime}\right)^{*}, \quad \xi^{\prime}=\left(\xi_{\alpha}^{\prime}\right)^{*}, \quad \xi^{\prime \prime}=\left(\xi_{\beta}^{\prime \prime}\right)^{*}, \quad u=\left(u_{s}\right)^{*}, \quad A_{1}=\left\|a_{v s}^{1}\right\|,
$$

$$
\operatorname{rang} A_{1}=r, \quad X(q), A_{1}(q) \subset C_{1} \quad \text { при } \quad q \in Q: t \geqslant 0, \quad|\xi|<\infty,
$$

$$
\xi=d \xi / d t, \quad|\xi|^{2}=\xi \cdot \xi=\sum_{v=1}^{n} \xi_{v}^{2}\left(\alpha=\overline{1, n_{1}}, \beta=\overline{1, n_{2}}, v=\overline{1, n}, \quad s=\overline{1, r}\right) \text {. }
$$

Допустимые управления $u(t)-$ кусочно-непрерывны на $T=[0, \infty)$ со значениями в $\omega^{1}=\omega^{1}(q) \subset E^{r}$ выпуклой компактной области

$$
0 \leqslant h_{1}(u, q) \leqslant \mathrm{Q}=\text { const }\left(h_{1}(u, q) \subset C, u \in E^{r}, q \in Q, \varrho>0\right) \text {, }
$$

где скалярная функция $h_{1}(u, q)$ принадлежит классу выпуклых функций $G$

$$
\begin{array}{r}
h_{1}\left(\alpha_{0} u\right)=\alpha_{0} h_{1}(u), h_{1}\left(u_{2}\right) \geqslant h_{1}\left(u_{1}\right)+\left(u_{2}-u_{1}\right) \nabla h_{1}\left(u_{1}\right)\left(\alpha_{0} \geqslant 0, u_{1} \neq 0\right), \\
h_{1}(0, q) \equiv 0, h_{1}(u, q)>0, u \in E_{0}^{r}=E^{r} \backslash 0, h(u, q) \subset C_{2} \text { на } E_{0}^{r} \times Q .
\end{array}
$$

Граница $\left\{h_{1}\left(u^{1}\right)=\varrho \mid u^{1}\right\}-$ замкнутая поверхность при $q=q_{0}=$ const.

Если $\varrho \rightarrow \infty$, то $\left|u^{1}\right| \rightarrow \infty$, и если $\varrho \rightarrow 0$, то $\left|u^{1}\right| \rightarrow 0$. Неравенство (1.2) - агрегат ограничений $a_{s}^{0}(q) \leqslant u_{s} \leqslant a_{s}^{1}(q)$, область $\omega^{1}-$ гладкая аппроксимация этого гиперкуба, регулярная [ $\left.{ }^{1}\right]$ при $r>1$. Обозначим $h_{1}^{0}(q)=\min _{e} h_{1}(e, q), h_{1}^{1}(q)=\max _{e} h_{1}(e, q), e=u|u|^{-1}$. Для задач с 
границей области $\omega^{1}$ в пределах $m_{0} \leqslant\left|u^{1}\right| \leqslant m_{1}$ достаточно выбрать $h_{1}(u, q)$ согласно условиям

$$
\varrho m_{1}^{-1} \leqslant h_{1}^{0}(q) \leqslant h_{1}^{1}(q) \leqslant \varrho m_{0}^{-1}\left(0<\text { const }=m_{0} \leqslant m_{1}=\text { const }\right) .
$$

Далее допустимы те из $\{u(t)\}$, которые приводят $\xi^{\prime}$-компоненту $\xi$ на множество цели $\xi_{\alpha}^{\prime}=0$ за конечное или бесконечное время $t_{1}-t_{0}$. Здесь $t_{1}\left(q_{0}\right)$ - первый момент примыкания $\xi^{\prime}\left(t, \xi_{0}\right) \rightarrow 0$ при $t \rightarrow t_{1}^{0}-0$, область значений $\xi_{0} \equiv \xi\left(t_{0}\right)$ управляемости определим ниже. Введем обратимое преобразование координат $x_{v}=x_{v}(q)$

$$
\begin{gathered}
x=\left(x_{v}\right)^{*}=\left(y_{i}, \gamma_{j}\right)^{*}, \quad x_{v}(t, 0) \equiv 0, \quad J=\left\|\frac{\partial x_{v}}{\partial \xi_{\sigma}}\right\|, \quad \operatorname{det} J \neq 0, \quad \xi^{\prime} \neq 0, \\
y=\left(y_{i}(q)\right)^{*}, \quad \gamma=\left(\gamma_{j}(q)\right)^{*}, \quad l=\operatorname{dim} y \ll n \quad(i=\overline{1, l, j} \overline{=1, n-l),} \\
\xi_{\alpha}^{\prime}=\xi_{\alpha}^{\prime}(t, x), \quad \xi_{\beta}^{\prime \prime}=\xi_{\beta}^{\prime \prime}(t, x), \quad \xi \sigma(t, 0) \equiv 0 \quad\left(\sigma, v=\overline{1, n}=n_{1}+n_{2}\right),
\end{gathered}
$$

чтобы использовать в качестве переменных агрегации $y(q)$ известные для системы (1.1) при $u \equiv 0$ информативные переменные $\left[{ }^{1-3}\right]$. Последними могут служить инварианты и $y$-автономные функции Ляпунова $y_{i}(q)$ со следующими свойствами.

Пусть $y_{i}(q), \gamma_{j}(q) \subset C$ на $Q$, в области $Q_{0}: t \geqslant 0, \quad \xi^{\prime} \in E^{n_{1}}, \xi^{\prime} \neq 0$, $\xi^{\prime \prime} \in E^{n_{2}}$, они принадлежат $C_{2}$. Примем, что $y_{i} \geqslant 0, y_{i}(q)$ неограничены на $Q$, а равенство $y=0$ эквивалентно $\xi^{\prime}=0$. Обозначим через $\Gamma$ и $E_{+}^{l}$ области значений $\gamma_{j}(q)$ и $0 \leqslant y_{i}(q)<\infty$. Тогда $x(q) \subset C$ на $R=T \times E_{+}^{l} \times \Gamma$ и $x(q) \subset C_{1}$ на $R_{0}=T \times E_{0}^{l} \times \Gamma$, где $E_{0}^{l}=E_{+}^{l} \backslash 0$. Пусть в $Q, R$ выполняются неравенства, содержащие некоторые непрерывные, положительно определенные функции $W_{a b}, a, b=\overline{1,2}\left(W_{11} \rightarrow \infty\right.$ при $\left|\xi^{\prime}\right| \rightarrow \infty$ и $W_{12} \rightarrow \infty$ при $\left.|y| \rightarrow \infty\right)$ со свойствами

$$
\begin{gathered}
W_{11}\left(\xi^{\prime}\right) \leqslant|y(q)| \leqslant W_{21}\left(\xi^{\prime}\right), \\
W_{12}(y) \leqslant\left|\xi^{\prime}(z)\right| \leqslant W_{22}(y) \quad\left(z=\left(t, x_{v}\right)^{*}, W_{a b}(0)=0\right) .
\end{gathered}
$$

Из (1.6) имеем $\xi^{\prime} \rightarrow 0,\left|\xi^{\prime}\right| \rightarrow \infty$ лишь при $y^{\prime} \rightarrow 0,|y| \rightarrow \infty$. Для простоты допустим, что $\left\{\xi^{\prime \prime}\right\}=E^{n_{2}}$ при $\gamma \in \Gamma$ и любых фиксированных $t_{0} \in T, y_{0} \in E_{+}^{l} . \quad$ В переменных $z=\left(t, y_{i}, \gamma_{j}\right)^{*}$ из (1.1), (1.5) и (1.6) получаем на $R_{0}$ систему, эквивалентную исходной

$$
\begin{gathered}
x=F(z)+M_{1}(z) u(F(t, 0) \equiv 0, u \in \omega: 0 \leqslant h(u, z) \leqslant \mathrm{Q}), \\
F=\left(Y_{i}^{1}, Z_{j}\right)^{*}, Y^{1}=\left(Y_{i}^{1}\right)^{*}, Z=\left(Z_{j}\right)^{*}, Y_{i}^{1}=X\left[y_{i}\right], Z_{j}=X\left[\gamma_{j}\right], \\
X[f]=\frac{\partial f}{\partial t}+X_{v} \frac{\partial f}{\partial \xi_{v}}, M_{1}=J A_{1}=\| \begin{array}{c}
P \\
L_{1}
\end{array}, \quad \operatorname{rang} M_{1}=r, \\
P=\left\|p_{i s}\right\|, p_{i s}=a_{v s}^{1} \frac{\partial y_{i}}{\partial \xi_{v}}, L_{1}=\left\|l_{j s}^{1}\right\|, l_{j s}^{1}=a_{v s}^{1} \frac{\partial \gamma_{j}}{\partial \xi_{v}}\left(h=h_{1}(u, q(z)) .\right.
\end{gathered}
$$

Здесь автономна ее $y$-подсистема при $u \equiv 0$, а матрица $A_{1}$ выбрана из условий

$$
\begin{aligned}
& \operatorname{rang} A \geqslant 1, A=\lambda^{-1} P, \lambda=\lambda(z) \geqslant W(y)>0(W(0)=0), \\
& \sup _{u_{1}} a \cdot u_{1}=G(p, y), u_{1} \in\{u \mid h(u, z)=1\} \quad\left(a=-A^{*} p\right)
\end{aligned}
$$


в области $D^{1}: t \geqslant 0, y \in E_{0}^{l},|y|<d_{1}, \gamma \in \Gamma(y \neq 0)$, где $\lambda, W \subset C_{1}$, по определению $y_{i}(q)$ имеем

$$
y=\lambda(z) Y(y)+P(z) u \quad\left(Y^{1}=\lambda Y(y)\right),
$$

$P=\lambda A=\lambda\left\|a_{i s}\right\|, Y_{i}(y), a_{i s}(z) \subset C_{1}, z \in D^{1}\left(W(y) \subset C, y \in E_{+}^{l},|y|<d_{1}\right)$.

Норм-инвариантные $\left[{ }^{1,2}\right]$, циклические $\left[{ }^{4}\right]$ и норм-автономные $\left[{ }^{5}\right]$ системы имеют информативные переменные $x$. Примем, что непрерывно дифференцируемые на $R_{0}$ величины $Y^{1}, Z, M_{1}$ удовлетворяют условиям, при которых для любой $x_{0} \equiv x\left(t_{0}\right) \in R_{0}$ и допустимого $u(t) \in \omega(z)$ решения (1.7) существуют, единственны и $\gamma$-продолжаемы $\left[{ }^{6}\right]$ в $R_{0}$. Из условий (1.5) и (1.6) следует $\left[{ }^{3}\right]$, что регулятору $u(z)$ системы (1.7), приводящему точки $D_{0}: t \geqslant 0, y \in E_{0}^{l} \cdot|y| \leqslant d^{0}, \gamma \in \Gamma$ на $y=0$, соответствует допустимый регулятор $u^{\prime}(q) \equiv u[z(q)]$ системы (1.1). Регулятор $u^{\prime}$ приводит точки $H_{0}: t \geqslant 0,0<\left|\xi^{\prime}\right| \leqslant h^{0}, \xi^{\prime \prime} \in E^{n_{2}}$ на $\xi^{\prime}=0$, где $h^{0}$ находим по $d^{0}$ из оценки

$$
W_{21}\left(\xi^{\prime}\right) \leqslant d^{0} \text { при } 0 \leqslant\left|\xi^{\prime}\right| \leqslant h^{0} \quad\left(d_{1}>d^{0}=\text { const, } h^{0}=\text { const }\right) .
$$

Если $u(z)$ приводит точки $R_{0}$ на $y=0$, то имеем $\xi^{\prime}$-стабилизацию в целом решения $\xi=0$ при $u^{\prime}(q) \equiv u[z(q)]$. Поэтому достаточно решить оптимальную задачу $y$-стабилизации для нахождения регулятора оптимальной $\xi^{\prime}$-стабилизации.

2. Рассмотрим в информативных переменных $x(q)$ задачу $\xi^{\prime}$-стабилизации $\xi=0$ при условии минимума функционала

$$
\begin{gathered}
I=\int_{t_{0}}^{t_{1}} \lambda(z) f(h, y) d \tau\left(f(\varrho, y)>0, y \in E_{0}{ }^{l},|y|<\infty\right), \\
h=h(u, z) \in G, 0 \leqslant h \leqslant \varrho, f(h, y) \subset C, y \in E_{+}{ }^{l} .
\end{gathered}
$$

Оптимальный регулятор $u_{0}(q) \equiv u^{0}[z(q)]$ найдем для допустимой функции $s=\min I[z, u]$ вида $s(y)$, которая непрерывна, положительно определена в $D_{1}$ и непрерывно дифференцируема на $D: y \in E_{0}^{l},|y|<$ $<d_{1}\left(D=D_{1} \backslash 0\right)$. Из (1.9) и $(2.1)$ имеем выражение гамильтониана $[4,6,7]$

$$
\begin{gathered}
B[s, u]=\lambda(L(u, z, p)+p \cdot Y), L=h a^{1} \cdot u_{1}+f(h, y), \\
p=\partial s / \partial y=\left(p_{i}\right)^{*}, a^{1}=-a=A^{*}(z) p, u_{1}=h^{-1} u(i=1, l \ll n) .
\end{gathered}
$$

В силу (1.3) вспомогательные управления $u_{s}^{1}$ связаны равенством

$$
h\left(u_{1}, z\right)-1=0 \quad\left(u_{1}=\left(u_{1}^{1}, \ldots, u_{r}^{1}\right)^{*}\right) .
$$

Обозначим $k(h, z, p)=\inf L\left(u_{1}, h, z, p\right)$ на поверхности (2.3). Из равенства $\inf _{u} L=\inf _{h} k$ находим, что $\mu^{1}=a^{1} \cdot u_{1}$ минимально на направлении

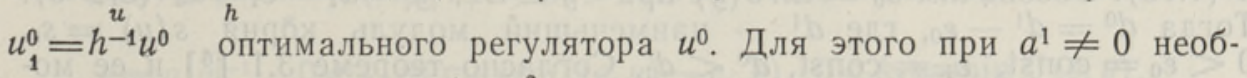
ходимо и достаточно, чтобы $u_{1}^{0}$ удовлетворял уравнениям

$$
a^{1}+\mu \partial h / \partial u_{1}=0, \quad h\left(u_{1}, z\right)=1 \quad\left(\mu=-i n f \mu^{1}>0\right) .
$$

Рассмотрим сопряженную $h(u, z)$ функцию $g(v, z)=\sup _{u_{1}} v \cdot u_{1}$ и обозначим $m^{*}=-g(v, z)$. Тогда имеем систему 


$$
v+m^{*} \partial h / \partial u_{*}=0, \quad h\left(u_{*}, z\right)=1 \quad\left(m^{*}<0\right) .
$$

Допуская существование достаточно гладкого решения $u *(v, z)$ системы (2.5), находим его, дифференцируя равенство $g(v, z)=v \cdot u *(v, z)$ с учетом $h\left[u_{*}(v, z), z\right] \equiv 1$, в виде

$$
u *(v, z)=\partial g / \partial v, \quad m^{*}(v, z)=-v \cdot \partial g / \partial v .
$$

Сопоставляя системы (2.4) и (2.6) при $v=v_{1} \in\{v \mid g(v, z)=1=$ $\left.=-m^{*}\right\}$, получаем значения экстремального направления $u^{0}$ и $\mu$ с учетом $(1.8)$

$$
u_{1}^{0}=\partial g / \partial v_{1}, \quad v_{1}=-\mu^{-1} a^{1}, \quad \mu=g\left(-a^{1}, z\right)=G(p, y) .
$$

Из определения $g(v, z)$ и свойств (1.3) находим, что $g(v)$ положительно однородна, выпукла, удовлетворяет при $v \equiv u$ всем условиям (1.3) $(g(u, z) \in G)$ и соотношениям

$$
\begin{array}{r}
g\left(\lambda_{0} v, z\right)=\lambda_{0} g(v, z), \quad g(0, z) \equiv 0, \quad g(v, z)>0, \quad v \neq 0 \quad\left(\lambda_{0} \geqslant 0\right), \\
h(u, z)=\sup _{v^{1}} u \cdot v^{1} \quad\left(v^{1} \in\{v \mid g(v, z)=1\}, \quad g(v, z)=\sup _{u_{1}} v \cdot u_{1}\right) .
\end{array}
$$

Функция $g(u, z)$ нецентрально строго выпукла по $u$ одновременно с функцией $h(u, z)$

$$
h\left(u_{2}\right)-h\left(u_{1}\right)>\left(u_{2}-u_{1}\right) \cdot \partial h / \partial u_{1} \quad\left(u_{2} \neq \alpha u_{1}, u_{1} \neq 0,0 \leqslant \alpha=\text { const }\right),
$$

для которой система (2.4) при $a^{1} \neq 0$ имеет единственное решение

$$
u_{1}^{0}=\partial g / \partial v_{1}=\partial g / \partial v . \quad\left(v_{*}=-a^{1}=-A^{*} p\right) .
$$

Для простоты примем, что $f(h, y)$ удовлетворяет по $h$ условию

$\mathrm{Q} f(h, y) \geqslant l(h, y) \equiv h(p \cdot Y+f(\varrho, y))-\varrho p \cdot Y \quad(y \in D, 0 \leqslant h \leqslant \varrho)$,

которое выполняется в задачах синтеза норм-автономных [5] систем. При (2.9) и (2.10) величина $k(h)$ достигает минимума в $h=\mathrm{Q}$. Оптимальный регулятор - вектор-функция $u^{0}(z)=\varrho u_{1}^{0}=\varrho \partial g / \partial a$. Здесь $a=-A^{*} p \neq 0$, если $p \neq 0$, и $A A^{*}>0 \sim \operatorname{rang} A=l$. Из $(1.7)-(1.9)$ и (2.2) получаем неравенства

$$
\begin{gathered}
B[s, u] \geqslant B\left[s, u^{0}\right] \quad \text { на } \quad D^{\prime} \times \omega(\omega: h(u, z) \leqslant \varrho), \\
s^{*}=-\lambda f(\varrho, y) \leqslant-W(y) f(\varrho, y) \quad\left(D^{1}: l \geqslant 0, \quad y \in E_{0}^{l},|y|<d_{1}, \quad \gamma \in \Gamma\right),
\end{gathered}
$$

если допустимая функция $s(y)$ удовлетворяет уравнению

$$
p \cdot Y(y)-\varrho G(p, y)+f(\varrho, y)=0 \quad\left(y \in E_{0}^{l},|y|<d_{1}, s(0)=0\right)
$$

и выполняется условие граничности (2.10). Следуя $\left[{ }^{6,7}\right]$, находим $d^{0}$ в (1.10). Обозначим $s_{0}=\inf s(y)$ при $y \in E_{+}^{l},|y|=d_{1}-\varepsilon_{1}=d_{0}\left(\varepsilon_{1}>0\right)$. Тогда $d^{0}=d^{1}-\varepsilon_{0}$, где $d^{1}-$ наименьший модуль корня $s(y)=s_{0}$, $0<\varepsilon_{0}=$ const, $\varepsilon_{1}=$ const, $d^{0}<d_{1}$. Согласно теореме 3.1 [ ${ }^{6}$ ] и ее модификации $\left[{ }^{3}\right]$ имеем следующий результат.

Если решение (2.12) - допустимая функция $s(y)$ и неравенство (2.10) справедливо, то для любых $z_{0}$ в цилиндре $C^{0}:\left|y_{0}\right| \leqslant d^{0}, y_{0} \in E_{0}^{l}$, граничный регулятор $u^{0}(z)=\varrho \partial g / \partial a$ является оптимальным по (2.1) регулятором $y$-стабилизации решения $x=0$ системы (1.7), (1.9).

Оптимальный регулятор $u_{0}(q)$ получаем из $u^{0}(z)$ подстановкой 
(1.5). Он приводит точки цилиндра $C_{1}^{0}: 0<\left|\xi_{0}^{\prime}\right| \leqslant h^{0}$ на $\xi^{\prime}=0$, если $h^{0}$ удовлетворяет (1.10). При выполнении условий леммы $1\left[{ }^{3}\right]$ время перехода $t_{1}-t_{0}$ конечно для точек $C_{1}^{0}$. Допустим, что динамическая система (1.7) при $u=u^{0}(z)$ имеет в области $D_{0}^{\prime}: t \geqslant 0$, $y \in E_{0}^{l},|y| \leqslant d^{\prime}, \gamma \in \Gamma$, функцию $V(z)$ со свойствами

$$
\begin{gathered}
V_{1}(y) \leqslant V(z) \leqslant V_{2}(y), \quad V(t, 0) \equiv 0, \quad V(t, 0, z) \equiv 0, \quad V_{1}(y)>0, \quad y \neq 0, \\
V^{\cdot}=-w(z) \leqslant-W(y), \quad V_{1} \rightarrow \infty, \quad|y| \rightarrow \infty, \quad V_{2}(0)=0 \\
\left(V(z), V_{a}(y) \subset C, \quad z \in D^{\prime}\right),
\end{gathered}
$$

при которых $y \rightarrow 0, t \rightarrow t_{1}-0$, если $z_{0} \in D_{0}: t \geqslant 0, y \in E_{0}^{l},|y| \leqslant d^{0}<d^{\prime}$, $\gamma \in \Gamma \quad\left(D_{0}^{\prime}=D^{\prime} \backslash 0\right)$. Обозначим $v_{21}=\max V_{2}|y| \leqslant d^{\prime}, v_{22}=\max V_{2}|y| \leqslant d^{0}$ $\left(y \in E_{+}^{l}\right)$. На интервале $\left[t_{0}, t_{1}\left(z_{0}\right)\right)$ имеем $V\left(t, x\left(t, z_{0}\right)\right) \equiv v(t) \rightarrow 0$ при $t \rightarrow t_{1}\left(z_{0}\right)-0$.

Л е м м а. Пусть для $V(z)$ известна непрерывная в области $D_{1}^{0}: t \geqslant 0$, $0<v<v_{21}$, функция $f(t, v)$, для которой

$$
f(t, V(z)) \geqslant w(z), \quad z \in D_{0}^{\prime},
$$

а неравенство $v * \geqslant-f(t, v)$ не имеет решений при $t_{0}, v_{0}$ из $D_{10}: t \geqslant 0$, $0<v \leqslant v_{22}$, лежащих в $D_{1}^{0}$ и примыкающих $\kappa v=0$ за конечное время $t_{1}-t_{0}<\infty$. Тогда решения (1.7) при $u=u^{0}(z) u z_{0} \in D_{0}$ примыкают к $у=0$ за бесконечное время.

Доказ ательство. Пусть справедливо обратное утверждение существует $x *\left(t, z_{0}\right)$ с конечным $\tau=t_{1}-t_{0}$. Рассмотрим $V\left(t, x_{*}\left(t, z_{0}\right)\right)=$ $=v_{*}(t)$ вдоль кривой $t, x_{*}\left(t, z_{0}\right)$, лежащей в области $D_{0}^{\prime}$, где выполнено равенство (2.13). В силу (2.14) получаем неравенство $v_{*}^{*}=-w \geqslant$ $\geqslant-f(t, v *)$, которое противоречит условиям (2.14).

Регулятор $u_{0}(q)$ дает оптимальную $\xi^{\prime}$-стабилизацию в целом, если $s(y)$ удовлетворяет (2.12) на $E_{0}^{l}, s \rightarrow \infty$ при $|y| \rightarrow \infty$ и условия (1.8), (1.9) и (2.10) выполняются в $R_{0}=T \times E_{0}^{l} \times \Gamma$.

Отметим следующие обстоятельства. Условие граничности и вид уравнения (2.12) можно аналогично получить для системы (1.1) общего типа (без информативных переменных). Для системы (1.1) с известными информативными переменными $x(q)$ выражения величин $h_{1}, X, A_{1}$ определяются на $h(u, z), F(z), M_{1}(z)$ и (1.7)-(1.9) с помощью преобразования (1.5). Задача синтеза для системы (1.1) в координатах $x(q)$ сводится к вопросу существования $A_{1}(q), f(h, y)$ и допустимой функции $s(y)$, удовлетворяющих (1.8), (2.10) и уравнению $(2.12)$ размерности $l \ll n$.

Используя $(2.8),(2.12)$, а также неравенства и обозначения

$$
\begin{gathered}
G(p, y) \geqslant|p| \min _{e} G(e, y), \min |p| \geqslant f(\varrho, y) k_{0}^{-1}(y)(0<f(\varrho, y) \sim p \neq 0), \\
e \equiv p|p|^{-1}, \quad 0<k_{0} \equiv \max _{e}(\varrho G(e, y)-e \cdot Y), 0<k_{1} \equiv \min _{e} G(e, y), \\
l_{0} \equiv 1-\varrho k_{1} k_{0}^{-1}, l_{1} \equiv k_{1} k_{0}^{-1}, \varphi(h, y) \equiv f^{-1}(\varrho, y) f(h, y),
\end{gathered}
$$

находим оценку, не содержащую $s, \varphi(h, y) \geqslant l_{1}(y) h+l_{0}(y)$, при которой выполняется условие граничности (2.10). 
Обозначим $\sigma=p \cdot Y$. Если допустимое решение (2.12) удовлетворяет оценке

$$
\sigma \geqslant \max _{h}\left[h\left(f(\varrho, y) \varrho^{-1}+\sigma \varrho^{-1}\right)-f(h, y)\right] \quad(0 \leqslant h \leqslant \varrho),
$$

то справедливо неравенство (2.10). Для вогнутой и линейной $f(h)$ на $[0$, e] условие $(2.15)$ имеет вид $\sigma \geqslant-f(0, y)$. Последнее достаточно также в случае выпуклой функции $f(h)$, удовлетворяющей оценке $|\partial f / \partial h|<\left|\varrho^{-1} f(\varrho, y)+\sigma\right|$ при $0 \leqslant h \leqslant \varrho, y \in E_{+}^{l}$.

3. Отметим два варианта задачи обращения в конструировании оптимального регулятора системы (1.1), (2.1). Сперва найдем условия существования решения задачи, близкой к проблеме Калмана-Летова $\left[{ }^{2,6}\right]$. Рассмотрим некоторую допустимую функцию $s(y)$, которая на $E_{+}^{l}$ удовлетворяет условиям

$$
\begin{gathered}
s(0)=0, s(y)>0, y \neq 0, p=\partial s / \partial y \neq 0, y \neq 0, s \rightarrow \infty|y| \rightarrow \infty, \\
s(y) \subset C, y \in E_{+}^{l}, s(y) \subset C_{1}, y \in E_{0}^{l} \equiv E_{+}^{l} \backslash 0 .
\end{gathered}
$$

Условия (3.1) выполняются, в частности, для $s(y) \in G=\{h(y)\}$. Относительно системы (1.1) предположим, что она имеет информативные переменные $x(q), \operatorname{rang} A=l \leqslant r=\operatorname{dim} u$, производная $d S / d \Theta \quad(d \Theta=$ $=\lambda d t)$ вдоль (1.9) при $u \equiv 0$ удовлетворяют неравенству

$$
\begin{gathered}
d S /\left.d \Theta\right|_{u \equiv 0}=p \cdot Y(y)<{ }_{\varrho} G(p, y) \quad\left(y \in E_{0}^{l}\right), \\
\lambda=\lambda(z) \geqslant W(y), \lambda(z) \subset C_{1} \quad y \neq 0\left(W(0)=0, W(y) \subset C \quad y \in E_{+}^{l}\right) .
\end{gathered}
$$

Возьмем $f^{1}(h, y)$ из класса непрерывных на $r_{0} \times E_{+}^{l}$ функций, удовлетворяющих там краевому условию и оценке

$$
\begin{gathered}
f^{1}(\varrho, y) \equiv \varrho G(p, y)-p \cdot Y(y)>0 \quad\left(y \in E_{0}^{l}\right), \\
f^{1}(h, y) \geqslant h G(p, y)-p \cdot Y(y)\left(h \in r_{0}: 0 \leqslant h \leqslant \varrho, y \in E_{0}^{l}\right) .
\end{gathered}
$$

Здесь и ниже используются обозначения (1.2), (1.5),(1.7)-(1.9). Для функции $\Phi^{1} \equiv f^{1}(h, y)-h G(p, y)$, достигающей минимума при $h=0$ или $h=\varrho$ (например, вогнутой или линейной по $h$ ), оценка (3.3) эквивалентна неравенству $f^{1}(0, y) \geqslant-p \cdot Y$. Согласно результатам $\left[{ }^{6,3}\right]$ находим, что $u^{0}(z)$ - оптимальный по (2.1) регулятор $y$-стабилизации системы (1.7) в целом, если выполняются условия (3.1)-(3.3) и $f(h, y) \equiv f^{1}(h, y)$. Требование $s^{*} \leqslant-W(y)$ здесь переходит в простое неравенство (3.2), которое выполняется, если $p \cdot Y<0$ при $y \in E_{0}^{l}$. Условия (3.2) и (3.3) эквивалентны дифференциальным неравенствам для $s(y)$ вида

$$
\max _{0 \leqslant h \leqslant \rho} F^{1}(h, y) \leqslant p \cdot Y<\varrho G(p, y) \quad\left(y \in E_{0}^{l}, F^{1} \equiv-\Phi^{1}\right),
$$

$$
f^{1}(\varrho, y) \equiv \varrho G(p, y)-p \cdot Y .
$$

Из (1.3) и (2.8) для функции $g(v, z)$ находим оценки $|v| h_{1}^{-1}(z) \leqslant g(v, z) \leqslant|v| h_{0}^{-1}(z)\left(h_{0}=\min _{e} h(e, z), h_{1}=\max _{e} h(e, z), \quad|e|=1\right)$.

Для задач с границей области управления в пределах $m_{0} \leqslant\left|u^{1}\right| \leqslant m_{1}$ необходимо и достаточно, чтобы выполнялись условия 


$$
\varrho m_{1}^{-1} \leqslant h_{0}(z) \leqslant h_{1}(z) \leqslant \varrho m_{0}^{-1}\left(z \in R=T \times E_{+}^{l} \times \Gamma\right) .
$$

Корни $\alpha_{1}, \ldots, \alpha_{l}$ уравнения $\operatorname{det}\left(A A^{*}-\alpha E\right)=0$ положительны в силу предположения rang $A=l$. Введем обозначения

$$
a_{0}^{2}(z)=\min _{i}\left\{\alpha_{i}\right\}, a_{1}^{2}(z)=\max _{i}\left\{\alpha_{i}\right\} \quad(i=\overline{1, l}) .
$$

В задаче с ограничениями (3.6) для выполнения условий (3.4) достаточно, чтобы выбор функции $f^{1}(h, y)$ и матрицы $A$ удовлетворял оценкам

$$
\sup _{0 \leqslant h \leqslant \rho}\left(m_{1} \varrho^{-1} a_{1}(z)-f^{1}(h, y)|p|^{-1}\right) \leqslant p^{0} \cdot Y(y)<m_{0} a_{0}(z)\left(p^{0}=\left|p^{-1}\right| p\right),
$$

которые находим из (3.5) и (3.7). Выберем $f^{1}(h, y)$ согласно (3.8). Если множество $m_{0}^{-1}|Y|<a_{0}(z)$ содержит подобласть $0<|y| \leqslant \varepsilon_{1}=$ $=$ const, $y \in E_{0}^{l}$, то получаем утверждение о локальной оптимальной $y$-стабилизации. Здесь условие $s(y) \rightarrow \infty$ при $|y| \rightarrow \infty$ отпадает. Заметим, что строгое неравенство в (3.8) принимает вид $p \cdot Y<0$, если rang $A<l$.

Рассмотрим новый вариант задачи обращения - с помощью аппроксимации области управления с границей в пределах $m_{0} \leqslant\left|u^{1}\right| \leqslant m_{1}$. Примем для простоты, что $\operatorname{rang}\left\|a_{i k}\right\|=l(i, k=\overline{1, l)}$. В классе $G$ возьмем функцию $g^{1}(u, y) \in G, g^{*}(v, z) \equiv g^{1}\left(C_{0}^{-1} v, y\right), \quad$ удовлетворяющую неравенствам

$$
\begin{gathered}
m_{0 Q_{0}^{-1}} \leqslant \min _{e}\left(\left|C_{0} e\right|^{-1} g^{1}(e, y)\right) \leqslant \max _{e}\left(\mid C_{0} e^{-1} g^{1}(e, y)\right) \leqslant m_{1} \varrho_{0}^{-1}, \\
e=v|v|^{-1}, \quad y \in E_{0}^{!} \quad C_{0}=\left\|-A^{*}, N\right\|, N=\left\|{ }_{E_{r-l}^{r-l}}^{0}\right\|
\end{gathered}
$$

и тождеству $g^{1}(p, 0, y)=G(p, y)(r \geqslant l)$, где $G(p, y), \quad 0<f\left(\varrho_{0}, y\right)$ заданные функции, для которых интегрируемо $\left[{ }^{8-10}\right]$ уравнение Якоби

$$
p \cdot Y(y)+f\left(\varrho_{0}, y\right)-\varrho_{0} G(p, y)=0 \quad\left(\varrho_{0}=\text { const }>0, y \in E_{0}^{l}\right)
$$

и полный интеграл $S(y, \alpha)$ является при $\alpha=\alpha^{0}=$ const допустимой функцией $s(y)\left(\alpha=\left(\alpha_{i}\right)^{*}, s(0)=0\right)$. Здесь неособенная $r \times r$ матрица $C_{0}, m_{0}=$ const $>0, m_{1}=$ const $>0, m_{0} \leqslant m_{1}-$ заданные числа.

Из (2.8) и (3.9) получаем соотношение

$$
\begin{gathered}
h^{*}(u, z)=\sup _{v_{1}} u \cdot v_{1}, v_{1} \in\left\{g^{*}(v, z)=1\right\} \quad\left(m_{0} \leqslant\left|u_{1}\right| \leqslant m_{1}\right), \\
\left.g^{*}\right|_{v=a} \equiv g^{1}\left(C_{0}^{-1} a, y\right) \equiv G(p, y)\left(p=\frac{\partial s}{\partial y}, h^{*}(u, z) \leqslant \varrho_{0}\right) .
\end{gathered}
$$

Пусть $s(y)$ - бесконечно большая функция, удовлетворяющая в области $0 \leqslant h^{*} \leqslant \varrho_{0}, y \in E_{+}^{l}, y \neq 0$, условию (2.10). Тогда при аппроксимации (3.11), произведенной $g^{1}(u, y)$, регулятор

$$
u_{*}^{0}(z)=\varrho_{0} \partial g^{*} / \partial a\left(a=-A^{*} p, y \in E_{0}^{l}\right)
$$

дает оптимальную по (2.1) $y$-стабилизацию в целом.

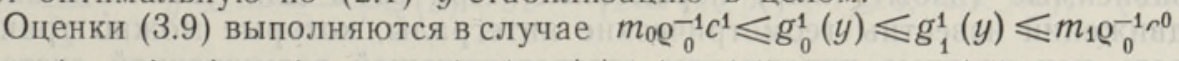
где $\quad g_{0}^{1}=\min _{e} g^{1}, \quad g_{1}^{1}=\max _{e} g^{1} \quad\left(e=|u|^{-1} u\right), \quad\left(c^{0}\right)^{2}=\min c_{s},\left(c^{1}\right)^{2}=\max c_{s}$, 
а $c_{s}$ - положительные корни $\operatorname{det}\left\|C_{0} C_{0}^{*}-c E\right\|=0, s=\overline{1, r}$. Можно показать, что $h^{*}(u, z) \equiv h_{*}(u, y)$, если $A A^{*} \equiv A_{2}(y)$. Выражая $f(\varrho, y)$ из равенства (3.10), нетрудно получить комбинацию обоих вариантов задачи обращения.

Условия примыкания решений динамической системы $\Sigma$ вида $x=$ $=X(x),\left(t, x_{v}\right)^{*} \in D:|t|<\infty,|x|<\infty, x \equiv Z(v=\overline{1, n})$, которая имеет $r$ функционально независимых, дифференцируемых на $E^{n} \backslash Z$ частных инвариантов

$$
\begin{gathered}
h_{s}(x)=0, h_{s}(0)=0, h_{s}(x) \subset C, x \in E^{n} \quad(s=1, r \leqslant n-1), \\
h=\Phi(x, h), \Phi(x, 0) \equiv 0, x \in E^{n \backslash Z}\left(h=\left(h_{s}\right)^{*}\right),
\end{gathered}
$$

выражает

Т еорем а. Пусть для $\Sigma$ известна непрерывная на $E^{n}$ и дифференцируемая вне $Z$ функция $V(x)$, которая в $H: h(x)=0$ имеет на $Z$ собственный минимум $V(P)>V(Z)=V(0)={ }^{\prime} 0, \quad$ и множества $Z: x \in H, V(x)=0, P: x \in H, x \equiv Z(x=0 \in Z)$ не пусты.

Пусть ограничено множество $L: x \in H, V(x)<l=$ const $(l>0)$ ина $R=L \cap P$ имеем $V \equiv X[V] \leqslant 0$.

Тогда любое решение системы для $x_{0} \equiv x\left(t_{0}\right) \in R$ примыкает $\kappa$ множеству $N=Z \cup Q$, где $Q-$ максимальное инвириантное множество, содержащееся в пересечении $L_{0}: V^{\cdot}(x)=0, V(x)<l, x \in H$.

Доказ а т ельство. Рассмотрим решение $x\left(t, x_{0}\right), x_{0} \in R$. Из ограниченности $L$ и неравенств $V\left(x\left(t, x_{0}\right)\right) \leqslant V\left(x_{0}\right)<l$ в области определения $T_{1}:\left[t_{0}, t_{1}\right)$ решения имеем $\left|x\left(t, x_{0}\right)\right| \leqslant r_{0}(l)<\infty$. Так как $x\left(t, x_{0}\right)$ не уходит в бесконечность при $t \in T_{1}$, то время $t_{1}-t_{0}$ определения решения в $R$ бесконечно или конечно. Для $t \rightarrow+\infty$ продолжаемого в $R$ решения $x\left(t, x_{0}\right)$ нет конечного $t_{1}$ примыкания $x\left(t, x_{0}\right) \rightarrow Z$ при $t \rightarrow t_{1}-0$. Тогда из непрерывности $V(x), h(x)$ получаем, что $\omega\left(x_{0}\right) \equiv \omega_{0}-$ предельное множество $x\left(t, x_{0}\right)$ - лежит во множестве $H \cap Z \cup V(x)=\lim _{t \rightarrow+\infty} V\left(x\left(t, x_{0}\right)\right)=l_{0}<l \quad\left(l_{0} \geqslant 0\right) . \quad$ Для $l_{0}=0 \quad$ имеем $x\left(t, x_{0}\right) \rightarrow Z$ при $t \rightarrow+\infty$. При $l_{0} \geq 0$ из инвариантности $\omega_{0}$ следует $V^{\cdot}\left(\omega_{0}^{\prime}\right)=0$ для всех $\omega_{0}^{\prime} \in \omega_{0}, \omega_{0}^{\prime} \equiv Z$.

Для $x\left(t, x_{0}\right)$ с конечным временем определения в $R$ есть первый момент $t_{1}$, когда $x \rightarrow Z$ при $t \rightarrow t_{1}-0$. Объединяя оба случая, получаем доказательство теоремы.

При $H \equiv E^{n}$ имеем отсюда модификацию теоремы VI [11] в случае недифференцируемых на $V(x)=0$ функций $V(x), X(x)$.

Если $H-$ произвольное инвариантное множество, где $V(H) \neq 0$, то утверждение теоремы сохраняется.

Следствие 1. Пусть условия теоремы выполняются для любого конечного $l>0$ и $V \rightarrow \infty$ при $|x| \rightarrow \infty$. Тогда любое решение $x\left(t, x_{0}\right)$ с $x_{0} \in P$ примыкает к $Z \cup Q_{1}$, где $Q_{1}$ - максимальное инвариантное множество в $V^{\cdot}(x)=0, x \in H$.

След ст ви е2. Если $\varrho(Z, N \backslash Z)=\varepsilon>0$ и $N \backslash Z=\varnothing$, то существует $\delta$-окрестность $Z(\delta>0)$, в которой все решения $x\left(t, x_{0}\right)$ $\left(x_{0} \in S(Z, \delta), x_{0} \equiv Z\right)$ примыкают к $Z$. В новых координатах $\zeta=\left(\xi \alpha, \eta_{s}\right)^{*}$, полученных гладким обратимым преобразованием $\xi_{\alpha}=\xi_{\alpha}(x), \quad \eta_{s}=$ $=h_{s}(x)(\alpha=1, \overline{n-r})$, согласно теореме находим $\xi\left(t, \zeta_{0}\right) \rightarrow \xi(N)$ при $t \rightarrow t_{1}-0$ и $\eta_{0}=0$, где $N=Z \cup Q$. В приложениях нередко используют зависимые (избыточные) переменные. При этом для установившихся движений известны геометрические интегралы, которые можно трактовать как частные инварианты соответствующей возмущенной системы вида $x=X(x), X(0)=0$. 
4. Применим полученные результаты в задачах обращения аппроксимацией для $y$-одномерной и диссипативной динамических систем.

4.1. Пусть переменные $y_{i}=y_{i}(q)$ - инварианты системы (1.1) при $u \equiv 0 \quad\left(Y_{i}^{1} \equiv 0\right)$ и в $D^{1} \times \omega$ выполняются условия

$$
\operatorname{det}\left\|a_{i k}(z)\right\| \neq 0, \operatorname{dim} y \equiv l \leqslant \operatorname{dim} u \equiv r \quad(i, k=\overline{1, l)},
$$

$$
\lambda \equiv 1, g^{1}(v, y) \equiv\left(g_{s}^{2} v_{s}^{2}\right)^{1 / 2}, \quad g^{*}(v, z) \equiv g^{1}\left(C_{0}^{-1} v, y\right), G^{2}(p, y)=g_{i}^{2} p_{i}^{2}, ~ \cdot
$$

$$
\begin{gathered}
h^{*}(u, z) \equiv \sup _{v^{1}} u \cdot v_{1}, v_{1} \in\left\{v \mid g^{*}(v, z)=1\right\}, g_{i}=g_{i}\left(y_{i}\right)>0, g_{\sigma}=g_{\sigma}(y)>0, \\
\partial g_{i} / \partial y_{s} \equiv 0, s \neq i, f^{2}(\varrho, y) \equiv \varrho^{2} \sum_{i=1}^{l} f_{i}^{2}\left(y_{i}\right), \partial f_{i} / \partial y_{s} \equiv 0, s \neq i,
\end{gathered}
$$

$$
\begin{gathered}
f_{i}>0, f^{2}\left(h^{*}, y\right) \geqslant h^{* 2} \sum_{i=1}^{l} f_{i}^{2}\left(y_{i}\right) \quad(s=\overline{1, r}, \sigma=\overline{=l+1, r)}, \\
I_{i}(\varepsilon)=\int_{\varepsilon}^{y_{i}} h_{i}(\tau) d \tau, \lim _{\varepsilon \rightarrow+0} I_{i}(\varepsilon)=I_{i}<\infty \quad\left(h_{i}=f_{i}(\tau) g_{i}^{-1}(\tau)>0\right),
\end{gathered}
$$

где $g_{s}, f_{i}, f-$ достаточно гладкие функции, $C_{0}=\left\|-A^{*}, N\right\|-$ матрица $(r \times r)$. В силу (4.1) решение $(2.12)$ - допустимая функция

$$
s_{1}(y)=\sum_{i=1}^{l} I_{i}\left(y_{i}\right), s_{1}(0)=0, s_{1}>0, y \in E_{0}^{l},|y|<d_{1}
$$

и выполнено условие (2.10). Согласно (3.12), (4.1) и (4.2) регулятор

$$
u_{1}^{0}(z)=\varrho\left(\sum_{i=1}^{l} f_{i}^{2}\right)^{-1 / 2}\left(C_{0}^{-1}\right)^{*} v^{1}\left(v^{1}=\left(g_{1} f_{1}, \ldots, g_{l} f_{l}, 0, \ldots, 0\right)^{*}, \operatorname{dim} v^{1}=r\right),
$$

оптимальный в $C^{0}$, осуществляет $\xi^{\prime}$-стабилизацию в целом, если условия $(1.8),(1.9),(2.10),(2.12)$ и (4.1) выполнены в $R_{0}$ и все $I_{i} \rightarrow+\infty$ при $y_{i} \rightarrow \infty$.

4.2. Рассмотрим одномерный случай $y=y_{1}=y_{1}(q)$ при условиях

$$
\begin{gathered}
Y_{1}^{1}=\lambda(z) Y_{1}(y), P=\lambda(z) A(z), \quad\left\|-a_{1 s}(z)\right\|^{*}=a_{1}=-A^{*} \neq 0, \\
g\left(a_{1}, z\right)=g_{0}(y), g(v, z)=\sup _{u_{1}} u_{1} \cdot v\left(h\left(u_{1}, z\right)=1\right), 0 \leqslant h \leqslant \varrho, \\
g_{0}(y)-Y_{0}(y)>0, \\
f(h, y) \geqslant f_{0}\left(g_{0} h-\varrho Y_{0}\right)\left(g_{0}-Y_{0}\right)^{-1}\left(Y_{0}=\varrho^{-1} Y_{1}, f_{0}=\varrho^{-1} f(\varrho, y)\right),
\end{gathered}
$$$$
I_{2}(\varepsilon)=\int_{\varepsilon}^{y} f_{0}\left(g_{0}-Y_{0}\right)^{-1} d \tau, \quad I_{2}(\varepsilon) \rightarrow I_{2}<\infty, \quad \varepsilon \rightarrow+0 \quad(t \geqslant 0,0<y, \quad \gamma \in \Gamma) .
$$

В этом случае функция $s_{2}(y) \equiv I_{2}(y)$ - допустима, условие $(2.10)$ выполнено и граничный регулятор $u_{2}^{0}(z)=\varrho \partial g / \partial a_{1}$ является оптимальным регулятором $\xi^{\prime}$-стабилизации в целом. Последнее следует из уравнения $y^{\circ}=\lambda_{\varrho}\left(Y_{0}-g_{0}\right)$ и условий $(1.6), \quad(4.3), \lambda \geqslant W(y)>0, y \neq 0$.

4.3. Рассмотрим вопрос $z$-стабилизации решения $z=0$ системы $\dot{x}=\frac{\partial H}{\partial y}, \quad y=-\frac{\partial H}{\partial x}+Q(z)+b v \quad\left(z=\left(x_{v}, y_{v}\right)^{*}, t \geqslant 0,|z|<\infty\right)$,

$$
H=H_{1}(x)+H_{2}(z), \quad H_{1}(0)=0, \quad H_{1}>0, \quad x \neq 0, \quad H_{1} \rightarrow \infty,
$$




$$
\begin{aligned}
& |x| \rightarrow \infty, \quad \frac{\partial H_{1}}{\partial x} \neq Q, \quad y=0, \quad x \neq 0, \\
& \frac{\partial H_{1}}{\partial x}=\left.Q\right|_{z=0}, \quad H_{2}(x, 0) \equiv 0, \quad \frac{\partial H_{2}}{\partial y} \equiv 0, \quad y=0 \text {, } \\
& 0<B=\left\|\frac{\partial^{2} H}{\partial y_{i} \partial y_{j}}\right\| \quad(i, j=\overline{1, n}),
\end{aligned}
$$

$0<\mathrm{const} \leqslant \min \beta_{i}, \quad \operatorname{det}\left|B-\beta_{i} E\right|=0, \quad b=A(z) x, \quad 2 R=A+A^{*}>0$,

$$
w(z)=x^{\cdot} \cdot Q(z) \leqslant 0 \quad\left(Q(z), H_{1}(z), A(z) \subset C_{1}, H_{2}(z) \subset C_{2},|z|<\infty\right),
$$

управляемой одним рулем $v=\left(v_{1}\right)^{*}$, при условии минимума расхода на линейную комбинацию диссипации ш и затрат вида

$$
\begin{gathered}
I_{3}=\int_{t_{0}}^{t_{1}}\left(|b \cdot \partial H / \partial y| f(H,|v|)-\varrho^{-1} w(z) f(H, \varrho)\right) d \tau \\
\left|v_{\mid}^{\prime} \leqslant \varrho, \quad 0<f(H, \varrho) \subset C, \quad \varrho f(H,|v|) \geqslant\right| v_{\mid}^{\prime} f(H, \varrho) \quad(H \geqslant 0) .
\end{gathered}
$$

Условия (4.4) означают, что $H(x, y)$ строго выпукла по импульсам $y_{i}$, имеет в $z=0$ собственный абсолютный минимум и $H \rightarrow+\infty$ при $|z| \rightarrow \infty$. Для системы (4.4) допустимая функция $s_{3}(y)$ и оптимальный по (4.5) регулятор z-стабилизации в целом имеют вид

$$
s_{3}(y)=s_{3}^{0}(H)=\int_{0}^{H} \varrho^{-1} f(\tau, \varrho) d \tau, \quad v^{0}=-M \quad\left(s_{3}(0)=0\right) .
$$

Действительно, значения (4.6) удовлетворяют в $T \times E^{2 n}$ первому из неравенств (2.11). Величина $H^{*}$ при $v=v^{0}$ в силу (4.4) равна $w(z)-\varrho R \partial H / \partial y \cdot \partial H / \partial y \leqslant 0$ и всюду отрицательна вне плоскости $y=0$, которая не является инвариантным множеством: $d y / d t \mid=$ $=Q(x, 0)-\partial H_{1} / \partial x \neq 0(x \neq 0)$ вне $x=0$. Из теоремы БарбашинаКрасовского следует, что $v^{0}$ для (4.4) осуществляет $z$-стабилизацию в целом.

Заметим, что в полученных результатах глобальным условиям оптимального синтеза можно придать локальную форму. В п. 4.3 функция $\lambda(z)$ задана не обязательно $H$-положительно определенной и введена невязка на диссипацию. Ясно, что условие граничности $(2.10)$, используемое здесь для упрощения постановки задачи, не является существенным. Следует отметить, что класс функционалов (2.1) включает квадратичные при $h^{2}=P_{1} u \cdot u$ и $y=y_{1}=P^{1} \xi \cdot \xi$ и описывает затраты времени, импульса и энергии. В постановке нет требований $u_{0}(t, \xi) \rightarrow 0$ при $\xi \rightarrow 0, f\left(q, u_{0}\right) \rightarrow 0$ при $\xi \rightarrow 0$ и дополнительного условия $t_{1}=\infty$.

В рассуждениях не используется дифференцируемость функций $f, s$, $u_{0}, V$ на множестве цели. Применение вместо $s(y)$ информативной функции $y=y_{1}(q)$ для установления глобальной управляемости в пп. 4.2 и 4.3 нетрудно распространить на системы вида (1.6)-(1.9). Необходимость в этом определяется ситуацией, аналогичной пп. 4.2 и 4.3, где $s_{2}(y), s_{3}(y)$ - не обязательно бесконечно большие функции. Оптимальные управления $u_{0}[t]=u_{0}\left(t, \xi\left(t, \xi_{0}\right)\right)$ в рассматриваемой задаче синтеза оказываются непрерывными функциями на интервале $\left[t_{0}, t_{1}\right)$. 


\section{Л И Т Е Р А Т У РА}

1. А т а н с М., Ф ал б П., Оптимальное управление, М., 1968.

2. Л е то в А. М., Динамика полета и управления, М., 1969.

3. К ей с И., Изв. АН ЭССР, Физ. Матем., 24, 178 (1975).

4. Р у м янце в В. В., ПММ, 36, вып. 6, 966 (1972).

5. Кей С И. А., Изв. АН СССР, Механика твердого тела, № 4, 44 (1975),

6. Р у м я нцев В. В., ПММ, 34, вып. 3, 440 (1970).

7. К р а сов ски й Н. Н., В кн.: Малкин И. Г., Теория устоймивости движений, доп. 4, М., 1966.

8. Л урье А. И., Аналитическая механика, М., 1961.

9. Ч ер т к ов Р.И., Метод Якоби в динамике твердого тела, Л., 1960.

10. П а р с Л., Аналитическая динамика, М., 1971.

11. Л е фше ц С., Исследование устойчивости прямым методом Ляпунова, М., 1964.

Ннститут кибернетики

Академии наук Эстонской ССР
Поступила в редакцию

6/IV 1976

\section{KEIS}

\section{DIMENSIOONI VÄHENDAMISEST MÕNEDE DUNAAMILISTE SUSTEEMIDE OPTIMAALSE SUNTEESI OLESANNETES}

Vaadeldakse juhtimise seisukohast lineaarse $n$-mõõtmelise dünaamilise süsteemi optimaalse sünteesi ülesannet. Võrratussüsteemiga antud mittestatsionaarne juhtimispiirkond aproksimeeritakse ühe kitsenduse abil. Informatiivsete muutujatega süsteemi tarvis taandatakse ülesanne $l \ll n$ dimensionaalse Jacobi võrrandi lahendamisele ja juhtimismaatriksi valikule.

Esitatakse Kalman-Letovi probleemi pöördülesande lahendamise meetod, mis on saadud juhtimispiirkonna aproksimeerimise teel.

Optimaalse sünteesi ülesanne on lahendatud $y$-ühemõõtmelise, $y$-invariantse ja dissipatiivse süsteemi puhul.

\section{KEIS}

\section{DIMENSION REDUCTION IN OPTIMAL SYNTHESIS OF SOME DYNAMICAL SYSTEMS}

The optimal synthesis problem for $n$-dimensional system, linear on control, is considered. Control lies in a region approximating one corresponding to several constraints. The dimension of Jacobi equation is diminished $(l \ll n)$ for systems with informative variables via control matrix selection. The approximation of control region for solution of Kalman-Letov inverse problem is proposed. Optimal synthesis for $y$-one-dimensional, $y$-invariant and dissipating systems is obtained in the paper. 